\title{
Ballistic expansion of a dipolar condensate
}

\author{
Stefano Giovanazzi, ${ }^{1}$ Axel Görlitz, ${ }^{2}$ and Tilman Pfau, ${ }^{2}$ \\ ${ }^{1}$ Center for Theoretical Physics and College of Science, Polish Academy of \\ Sciences, Aleja Lotników 32/46, 02-668 Warsaw, Poland \\ 2 th Institute of Physics, University of Stuttgart, D-70550 Stuttgart, Germany. \\ E-mail: stevbolz@yahoo.it
}

\begin{abstract}
We have studied the free expansion of a Bose condensate in which both the usual s-wave contact interaction and the dipole-dipole interaction contribute considerably to the total interaction energy. We calculate corrections due to dipolar forces to the expansion of such a condensate after release from a trap. In the Thomas-Fermi limit, we find that the modifications are to lowest order independent from the total number of atoms.
\end{abstract}

PACS numbers: 03.75.Fi, 05.30.Jp

Submitted to: J. Opt. B: Quantum Semiclass. Opt.

\section{Introduction}

In the atomic Bose-Einstein condensates (BECs) realized so far, the atoms interact essentially only at very short distance (Van-der Waals interaction) compared to the separation between atoms. The only relevant parameter that characterizes the interaction is the s-wave scattering length [1]. Recently dipole-dipole interactions in atomic BECs have attracted increasing interest [2, 3, 4, 5, 6, 8, 8, 9, 10, 11. These interactions would largely enrich the variety of phenomena to be observed due to their long-range and anisotropic character.

Atoms like rubidium and sodium which have been successfully Bose-condensed, posses a magnetic dipole moment, but it is very small. We focus here on another atom as a possible candidate for the realization of a dipolar condensate, namely chromium. Chromium atoms have a larger magnetic moment $m=6 \mu_{B}$, where $\mu_{B}$ is the Bohr magneton, and interact with non negligible dipole dipole forces. These anisotropic forces compete with s-wave scattering. If the s-wave scattering length (unknown for chromium) is not too large, then dipolar forces could be observed directly through small but appreciable modifications in the ground state density distribution or even in the occurrence of instability.

We discuss in this paper the dipolar effects on the free expansion of a dipolar BEC. In many cases, trapped condensates are too small to obtain clear images in absorption imaging and therefore the condensate is released from the trap to expand in order to obtain larger dimension. Because the dipolar effects in chromium are expected to be not too large, it is interesting to maximize the dipolar effects in ballistic expansion by tuning external parameters like the trap anisotropy. 


\section{Magnetic dipole interaction}

We consider a dipolar gas of neutral atoms with magnetic moment $m$ oriented along the magnetic field

$$
\mathbf{B}(t)=B[\cos \varphi \hat{\mathbf{z}}+\sin \varphi(\cos (\Omega t) \hat{\mathbf{x}}+\sin (\Omega t) \hat{\mathbf{y}})] .
$$

The frequency $\Omega$ of the radial component of the magnetic field is chosen such that $\omega_{\text {Larmor }} \gg \Omega \gg \omega_{\text {trap }}$, where $\omega_{\text {trap }}$ is any of the trap frequencies. This corresponds to the assumption that the atoms are not moving during one rotation of the magnetic field and the magnetic moments will follow adiabatically the total magnetic field. The resulting long range part of the interatomic interaction is [11

$$
U_{\mathrm{dd}}(\mathbf{r})=-\frac{\mu_{0} m^{2}}{4 \pi}\left(\frac{3 \cos ^{2} \varphi-1}{2}\right)\left(\frac{3 \cos ^{2} \theta-1}{r^{3}}\right),
$$

where $\theta$ is the angle between the interatomic separation vector $\mathbf{r}$ and the $z$-axes. Here $m$ is the atomic magnetic moment and $\mu_{0}$ is the magnetic permeability of the vacuum. The angle $\varphi$ serves as a "knob" to tune the dipolar interaction in real time ( $\varphi$ may have a low frequency time dependence).

\section{Thomas-Fermi mean-field analysis}

Our analysis is based on the zero-temperature $(T=0)$ mean-field approach already used in the context of dipolar Bose gases [2, 2, 6. Such a description can be accomplished through the Gross-Pitaevskii equation [1] for the condensate order parameter $\Psi(\mathbf{r}, t)$ (normalized to the number of atoms $N$ )

$$
i \hbar \frac{\partial \Psi}{\partial t}=\left[-\frac{\hbar^{2} \boldsymbol{\Delta}}{2 M}+\frac{M \omega_{r}^{2}}{2}\left(x^{2}+y^{2}\right)+\frac{M \omega_{z}^{2}}{2} z^{2}+V_{m f}\right] \Psi,
$$

where $M$ is the atomic mass, $\omega_{r}\left(\omega_{z}\right)$ the radial (longitudinal) trap frequency and where the mean-field potential $V_{m f}$ is given by

$$
V_{m f}(\mathbf{r})=\frac{4 \pi \hbar^{2} a}{M}|\Psi(\mathbf{r})|^{2}+\int d \mathbf{r}^{\prime}\left|\Psi\left(\mathbf{r}^{\prime}\right)\right|^{2} U_{\mathrm{dd}}\left(\mathbf{r}-\mathbf{r}^{\prime}\right),
$$

where $a$ is taken to be a positive s-wave scattering length.

A measure of the strength of the dipole-dipole interaction relative to the s-wave scattering energy is given by the dimensionless quantity

$$
\varepsilon_{\mathrm{dd}}=\frac{\mu_{0} m^{2} M}{12 \pi \hbar^{2} a} \text {. }
$$

$\varepsilon_{\mathrm{dd}}$ is defined in such a way that when it is larger than one $\left(\varepsilon_{\mathrm{dd}}>1\right)$ a homogenous dipolar condensate is unstable against collapse in the Thomas-Fermi limit [6] and in the absence of an external potential.

For chromium the s-wave scattering length $a_{\mathrm{Cr}}$ is not known. If we assume that $a_{\mathrm{Cr}}$ is equal to the sodium scattering length $(a=2.8 \mathrm{~nm})$ the dipolar strength parameter becomes $\varepsilon_{\mathrm{dd}}^{\mathrm{Cr}}=0.29$ ( $m=6 \mu_{B}$, where $\mu_{B}$ is the Bohr magneton). In comparison, for rubidium and sodium the corresponding values of $\varepsilon_{\mathrm{dd}}$ are much smaller. In contrast, for heteronuclear molecules, we expect a much larger $\varepsilon_{\mathrm{dd}}$.

The starting point for our analysis is the limit of a large number of atoms, namely the Thomas-Fermi limit in which the kinetic energy has a very small effect on the ground state density distribution. This physically relevant case represents also an ideal situation from an experimental point of view because an observable quantity like the modification of the condensate shape is independent of the number of atoms. 


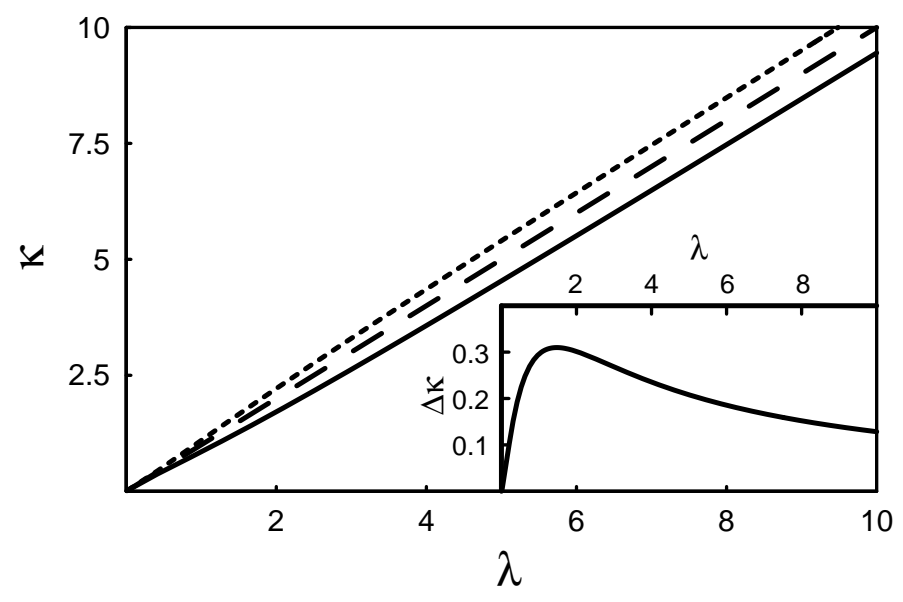

Figure 1. Equilibrium condensate anisotropy parameter $\kappa=\xi_{r} / \xi_{z}$ versus the trap anisotropy parameter $\lambda=\omega_{z} / \omega_{r} . \quad \kappa$ is calculated in the Thomas-Fermi regime with $\varepsilon_{\mathrm{dd}}=0.29$. The three curves correspond to three different values of $\varphi$ : the curve for $\varphi=0$ is solid, for $\varphi=54.7^{\circ}$ is dashed and for $\varphi=90^{\circ}$ it is dotted. The inset shows $\Delta \kappa=\left(\kappa\left(\varphi=90^{\circ}\right)-\kappa\left(\varphi=0^{\circ}\right)\right) / \kappa\left(\varphi=57^{\circ}\right)$ versus the trap anisotropy parameter $\lambda$.

\section{Condensate expansion}

The expansion of the condensate (as well as its equilibrium configuration) can be studied using a time-dependent mean-field variation approach similar to Ref. 22, 15] (see also [11]) by assuming a gaussian wave function as an ansatz

$$
\Psi(x, y, z, t)=\left(\frac{\pi^{-3 / 2} N}{\xi_{x} \xi_{y} \xi_{z}}\right)^{1 / 2} \prod_{\eta=x, y, z} e^{-\frac{\eta^{2}}{2 \xi_{\eta}^{2}}+i \eta^{2} \beta_{\eta}},
$$

where $\xi_{\eta}$ (width) and $\beta_{\eta}$ are time dependent variational parameters. It is easy to derive the following set of equation for the condensate widths

$$
\ddot{\xi_{\eta}}=-\frac{2}{M} \frac{\partial}{\partial \xi_{\eta}} \frac{H_{\mathrm{tot}}}{N}\left[\xi_{x}, \xi_{y}, \xi_{z}\right]
$$

$\left(\beta_{\eta}=\frac{M \dot{\xi}_{\eta}}{2 \hbar \xi_{\eta}}\right)$. The various contributions to the total mean-field energy functional $H_{\text {tot }}$ evaluated on the gaussian wave function are: (a) the kinetic energy in the ThomasFermi limit $H_{\text {kin }}=(N M / 4)\left(\dot{\xi}_{x}^{2}+\dot{\xi}_{y}^{2}+\dot{\xi}_{z}^{2}\right)$ (where we neglect here the contribution of the zero-point fluctuations $\left.\left(N \hbar^{2} / 4 M\right)\left(1 / \xi_{x}^{2}+1 / \xi_{y}^{2}+1 / \xi_{z}^{2}\right)\right)$; (b) the potential energy in the harmonic trap $H_{\mathrm{ho}}=(N m / 4)\left(\omega_{r}^{2} \xi_{x}^{2}+\omega_{r}^{2} \xi_{y}^{2}+\omega_{z}^{2} \xi_{z}^{2}\right)$; (c) the mean-field energy due to s-wave scattering

$$
H_{s}=\frac{N^{2} \hbar^{2} a}{m \sqrt{2 \pi}} \frac{1}{\xi_{r}^{2} \xi_{z}}
$$

(d) the mean-field magnetic dipole-dipole energy, which for a cylindrically symmetric condensate is given by

$$
H_{d d}=-\frac{N^{2} \mu_{0} \mu^{2}}{12 \pi \sqrt{2 \pi}} \frac{f\left(\xi_{r} / \xi_{z}\right)}{\xi_{r}^{2} \xi_{z}}
$$




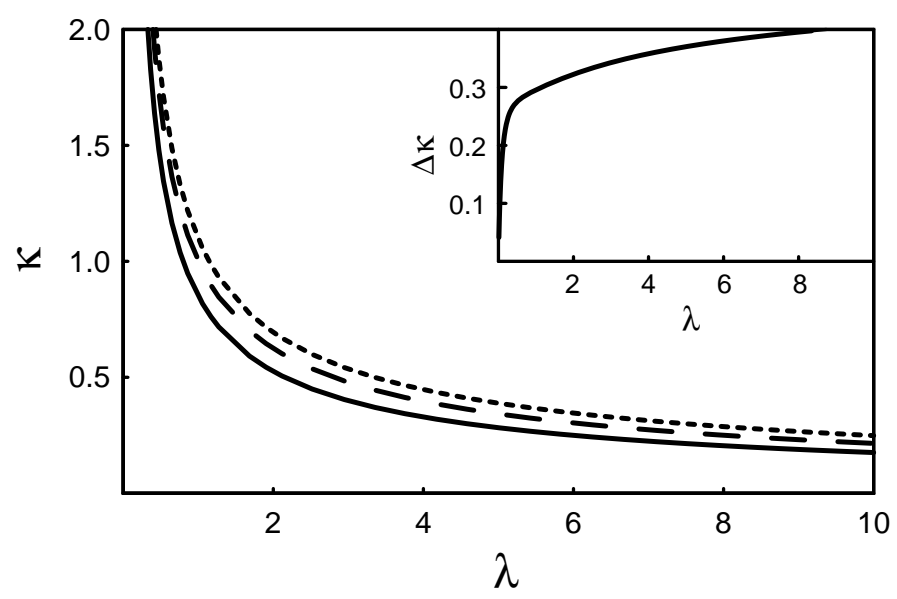

Figure 2. Condensate anisotropy parameter $\kappa$ after expansion versus the trap anisotropy parameter $\lambda . \quad \kappa$ is calculated in the Thomas-Fermi regime with $\varepsilon_{\mathrm{dd}}=0.29$. The three curves correspond to three different values of $\varphi$ : the curve for $\varphi=0$ is solid, for $\varphi=54.7^{\circ}$ is dashed and for $\varphi=90^{\circ}$ it is dotted. The inset shows $\left(\kappa\left(\varphi=90^{\circ}\right)-\kappa\left(\varphi=0^{\circ}\right)\right) / \kappa\left(\varphi=57^{\circ}\right)$ versus the trap anisotropy parameter $\lambda$. After expansion, the observability is improved in the "pancake" geometry (large $\lambda$ ).

$$
f(\kappa)=\frac{1+2 \kappa^{2}}{1-\kappa^{2}}-\frac{3 \kappa^{2} \tanh ^{-1} \sqrt{1-\kappa^{2}}}{\left(1-\kappa^{2}\right)^{3 / 2}}
$$

Here, we have introduced the radial width $\xi_{r}=\xi_{x}=\xi_{y}$. The argument of $f$ is the condensate aspect ratio $\kappa=\xi_{r} / \xi_{z}$.

The anisotropy of the static dipole-dipole interaction is directly manifest in the modification of the shape of the condensate. In the Thomas-Fermi limit the ratio

$$
\kappa=\frac{\left\langle x^{2}\right\rangle^{1 / 2}}{\left\langle z^{2}\right\rangle^{1 / 2}}=\frac{\xi_{r}}{\xi_{z}}
$$

of the radial $\left\langle x^{2}\right\rangle^{1 / 2}$ and longitudinal width $\left\langle z^{2}\right\rangle^{1 / 2}$ of the condensate is independent of the number of atoms, because the dipolar energy has the same scaling dependence as the s-wave scattering energy. In particular $\kappa$ varies as function of the "knob" angle $\varphi$. The anisotropy parameter $\kappa$ of the condensate can be studied for an equilibrium situation, in which the condensate is harmonically trapped, or after a free expansion. Generally, the condensate anisotropy $\kappa$ is a function of the trap anisotropy parameter

$$
\lambda=\omega_{z} / \omega_{r} .
$$

In the Thomas-Fermi limit $\kappa$ is a solution of the following equation

$$
\lambda^{2}=\kappa^{2} \frac{1-\varepsilon_{\mathrm{dd}} f(\kappa)-\varepsilon_{\mathrm{dd}} \kappa \frac{\partial f}{\partial \kappa}(\kappa)}{1-\varepsilon_{\mathrm{dd}} f(\kappa)+\frac{1}{2} \varepsilon_{\mathrm{dd}} \kappa \frac{\partial f}{\partial \kappa}(\kappa)} .
$$

It is straightforward to see the standard $\kappa=\lambda$ result for a trapped condensate if dipolar forces are absent. When $\varepsilon_{\mathrm{dd}} \ll 1$ we can evaluate analytically the variation of the aspect ratio $\kappa$ and the variation of the "condensate volume" $V=\left(\left\langle x^{2}\right\rangle\left\langle y^{2}\right\rangle\left\langle z^{2}\right\rangle\right)^{1 / 2}$

$$
\frac{\delta \kappa}{\kappa}=\frac{3}{4} \varepsilon_{\mathrm{dd}} \lambda \frac{\partial f}{\partial \kappa}(\lambda)
$$




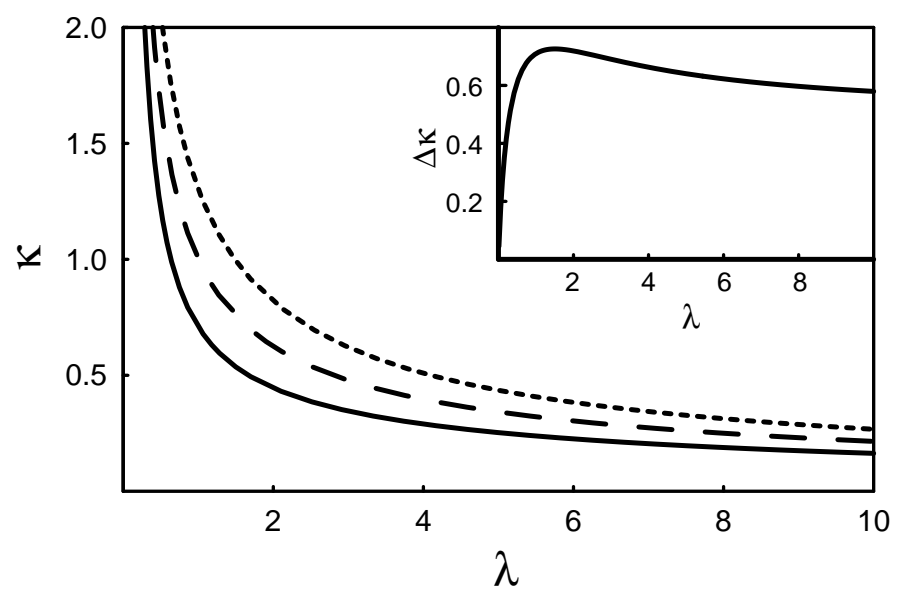

Figure 3. Condensate anisotropy parameter $\kappa$ after a free expansion with 'inverted' dipolar coupling. When the condensate is released, the angle $\varphi$ is switched from $\varphi=0$ to $\varphi=90^{\circ} . \kappa$ is calculated in the Thomas-Fermi regime with $\varepsilon=0.29$ as function of the trap anisotropy parameter $\lambda$. The three curves correspond to three different values of $\varphi$ : the curve for $\varphi=0$ is solid, for $\varphi=54.7^{\circ}$ is dashed and for $\varphi=90^{\circ}$ it is dotted. The inset shows $\left(\kappa\left(\varphi=90^{\circ}\right)-\kappa\left(\varphi=0^{\circ}\right)\right) / \kappa\left(\varphi=57^{\circ}\right)$ versus the trap anisotropy parameter $\lambda$.

$$
\frac{\delta V}{V}=-\varepsilon_{\mathrm{dd}}\left(\frac{3}{5} f(\lambda)+\frac{9}{20} \lambda \frac{\partial f}{\partial \kappa}(\lambda)\right) .
$$

The condensate's aspect ratio $\kappa$ is a more universal parameter than the overall size $V$ because it depends only on the trap geometry and not on the number of atoms. In particular the variation of $\kappa$ is easy to measure because the value of $\kappa$ in absence of dipolar forces is well know (in the Thomas-Fermi limit, it is given by the trap anisotropy $\lambda$; therefore $\delta \kappa=\kappa-\lambda$ ). Moreover it is possible to change the tuning angle $\varphi$ and measure the difference $\kappa\left(\varphi=90^{\circ}\right)-\kappa\left(\varphi=0^{\circ}\right)$. In contrast, the variation of the condensate volume $V$ is more difficult to measure because it requires the knowledge of the number of atoms, a quantity that is usually difficult to determine with sufficient accuracy. From Eq. (14) it is possible to see that $\kappa$ is not sensitive to the dipolar forces for high and in particular for small values of $\lambda$ because the product $\kappa \frac{\partial f}{\partial \kappa}(\kappa)$ goes to zero in the two limits $\kappa \rightarrow 0$ and $\kappa \rightarrow \infty$. The maximum linear response of $\kappa$ to $\varepsilon_{\mathrm{dd}}$, i.e. $\delta \kappa \sim .64 \varepsilon_{\mathrm{dd}}$, is obtained for $\lambda \approx 2$.

Figure 1 shows the equilibrium anisotropy parameter $\kappa$ of the condensate versus the trap anisotropy parameter $\lambda$ in the Thomas-Fermi regime, calculated for a chromium condensate where a s-wave scattering length of $a=2.8 \mathrm{~nm}$ is assumed $\left(\varepsilon_{\mathrm{dd}}=0.29\right)$. The effect of the dipole interaction is to reduce $\kappa$ for $\varphi=0^{\circ}$ or to increase $\kappa$ for $\varphi=90^{\circ}$ with respect to the equilibrium value of $\kappa$ in absence of dipolar forces (see Figure 1). Note that by varying $\varphi$, the total variation of $\kappa$ can be increased. Thus, the influence of the dipolar force becomes more visible.

Now we consider the expansion of the condensate. Like for the static properties, the evolution of the condensate anisotropy parameter is independent of the number of atoms in the Thomas-Fermi limit. Figure 2 shows the asymptotic values of the anisotropy parameter $\kappa(t=\infty)$ after expansion. In a finite time experiment these values are approximated by the ratios of the velocities $\dot{\xi}_{r} / \dot{\xi}_{z}$. 
Similar to the equilibrium case the effect of a (weak) dipole interaction is to reduce $\kappa$ for $\varphi=0^{\circ}$ (or to increase $\kappa$ for $\varphi=90^{\circ}$ ) with respect to the values $\kappa(t=\infty$ ) in absence of dipolar forces (see Figure 2). In the absence of dipolar forces $\kappa(t=\infty)$ is always inverted after expansion.

According to the results shown in Figure 2, a pancake trap will enhance the variation of $\kappa$ with $\varphi$. Assuming $a=2.8 \mathrm{~nm}$, the variation of $\kappa$ is $40 \%$. If $\varepsilon_{\mathrm{dd}}$ is in reality smaller than our assumption one should think of some scheme to amplify the effect. A simple scheme to do that is the following (see Fig. 3): when the condensate starts expanding, the sign of the effective dipolar coupling should be changed by changing the angle $\varphi$ from $0^{\circ}$ to $90^{\circ}$. With this simple technique the observability is further increased.

The particular case of a dipolar condensate initially trapped in an isotropic trap $(\lambda=1)$ deserves some consideration. When the condensate is released its anisotropy, i.e. its shape (cigar deformed for $\varphi=0^{\circ}$ and pancake deformed for $\varphi=90^{\circ}$ ), is conserved during the time evolution. Indeed the set of equations (7) with a general time-dependent isotropic harmonic potential frequency $\omega_{0}(t)$ admits a solution of the form $\xi_{\eta}(t)=\xi(t) \xi_{\eta}^{0}$ with $\eta=x, y, z$ (where $\xi_{\eta}^{0}$ for instance are stationary solution of (7) with $\left.\omega_{0}(t)=\omega_{0}\right)$. Similarly the frequency of the monopole mode in an isotropic trap is given by $\sqrt{5} \omega_{0}$, where $\omega_{0}$ is the trap frequency, irrespectively of the dipolar forces.

\section{Conclusions}

We have theoretically investigate the static properties as well the expansion of a dipolar condensate in the Thomas-Fermi regime. We found that the pancake geometry is a more suitable trap geometry to observe dipolar effects, since they are enhanced during expansion.

Note added in proof - We are aware of one similar work on the expansion of a dipolar condensate [16].

\section{Acknowledgments}

Funding was provided by the RTN network "cold quantum gases" under the contract number HPRN-CT-2000-00125, and the Deutsche Forschungsgemeinschaft. We thank Kazimierz Rzążewski for a critical reading of this manuscript.

[1] F. Dalfovo et al., Rev. Mod. Phys. 71, 463 (1999).

[2] S. Yi and L. You, Phys. Rev. A 61, 041604 (2000); S. Yi and L. You, Phys. Rev. A 63, 053607 (2001).

[3] K. Goral, K. Rzazewski, and T. Pfau, Phys. Rev. A 61, 051601 (2000); J.-P. Martikainen, Matt Mackie, and K.-A. Suominen, Phys. Rev. A 64, 037601 (2001).

[4] L. Santos et al., Phys. Rev. Lett. 85, 1791 (2000); L. Santos et al., Phys. Rev. Lett. 88, 139904 (2002).

[5] M.A. Baranov et al., cond-mat/0109437.

[6] K. Goral, L. Santos and M. Lewenstein, Phys. Rev. Lett. 88, 170406 (2002).

[7] H. Pu, W. Zhang, and P. Meystre, Phys. Rev. Lett. 87, 140405 (2001); W. Zhang, H. Pu, C. Search, and P. Meystre, Phys. Rev. Lett. 88, 060401 (2002).

[8] D. DeMille, Phys. Rev. Lett. 88, 067901 (2002).

[9] D. O'Dell, et al., Phys. Rev. Lett. 84, 5687 (2000); S. Giovanazzi, D. O’Dell, and G. Kurizki, Phys. Rev. A 63, 031603 (2001); S. Giovanazzi et al., Europhys. Lett. 56, 1 (2001); S. Giovanazzi, D. O’Dell, and G. Kurizki, Phys. Rev. Lett. 88, 130402 (2002).

[10] M. Baranov et al., cond-mat/0201100.

[11] S. Giovanazzi, A. Görlitz, and T. Pfau, Phys. Rev. Lett. 89, 130401 (2002). 
[12] J.D. Weinstein, et al., Nature (London) 395, 148 (1998).

[13] J. Weinstein, et al., Phys. Rev. A 57.3173 (1998).

[14] K. Goral, and L. Santos, cond-mat/0203542; S. Yi and L. You, cond-mat/0111256

[15] Y. Castin, and R. Dum. Phys. Rev. Lett. 77, 5315 (1996).

[16] S. Yi and L. You, cond-mat/0210677. 